\title{
Towards longwave infrared tuneable filters for multispectral thermal imaging applications
}

\author{
by H. Mao*, K. K. M. B. D. Silva*, M. Martyniuk*, J. Antoszewski*, J. Bumgarner* and L. Faraone* \\ *School of Electrical, Electronics and Computer Engineering, The University of Western Australia, 35 Stirling \\ Highway, Crawley 6009, Western Australia, 20878492@student.uwa.edu.au
}

\begin{abstract}
Many thermal imaging applications stand to gain from the inclusion of multispectral capabilities in the long wave infrared (LWIR). While infrared spectral imaging systems presently exist, the addition of multispectral capabilities will provide a number of advantages, including the ability to accurately quantify the temperature distribution a scene, and the potential for dramatically improved target identification using spectral processing algorithms. We report here on our microspectrometer technology, based on electrically tuneable Fabry-Perot filters, which is moving towards implementation in the LWIR (8 - 12 micron wavelength) for multispectral thermal imaging applications.
\end{abstract}

\section{Introduction}

State-of-the-art infrared (IR) focal plane array (FPA) technologies aim to improve the performance of IR imaging systems by reducing cooling requirements and/or adding so-called multi-colour capability, which allows on-pixel information to be gathered from two or more spectral regions. Spectral information allows improved target recognition and reduced false alarm rates in military applications, and accurate temperature determination in civilian applications. It has been recognised, however, that in order to extract spectroscopic or detailed information from a cluttered image, much better spectral resolution is required than can be afforded by such multi-colour systems. A number of research programs are aiming to address this issue by obtaining significantly better spectral resolution by developing technologies for the integration of individual tuneable optical filters with infrared imaging arrays. The approach chosen by The Microelectronics Research Group (MRG) at The University of Western Australia is to develop a micro-electromechanical systems (MEMS) technology that is compatible with large format two-dimensional infrared FPAs. Such a device structure consists of an electrostatically controlled Fabry-Perot filter that is integrated optically ahead of the detector array. This can consist of an individual spectrometer per pixel, or a large area filter covering the entire imaging array.

\section{Principle and implementation}

The MRG microspectrometer [1, 2], as illustrated in figure 1(a) and (b), consists of a MEMS tunable Fabry-Perot filter mounted optically ahead of an infrared photodetector. Electrostatic actuation allows the top mirror to be moved downwards in figure 1, thus decreasing the mirror spacing and, thereby, shifting the optical pass-band of the spectrometer. Each mirror in the microspectrometer is a multi-layer Bragg mirror structure consisting of three layers, germanium - silicon monoxide - germanium. A structural layer of silicon nitride supports the top mirror. This structural layer extends to the support arms that actuate the mirror structure. This fabrication process is horizontally scalable, making it compatible with implementation as a single filter covering the entire array, or as an array of filters on a per-pixel level at the focal plane, as depicted in figure 1 (c).

(a)

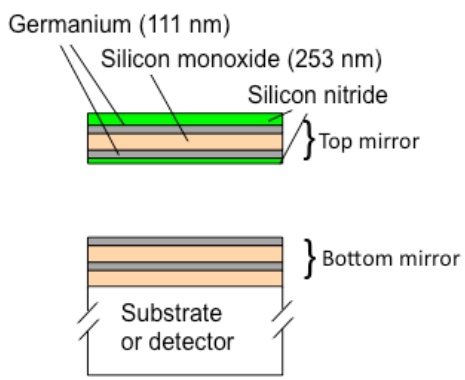

(b)

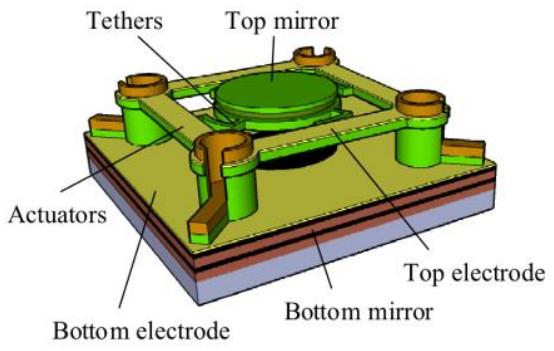

(c)

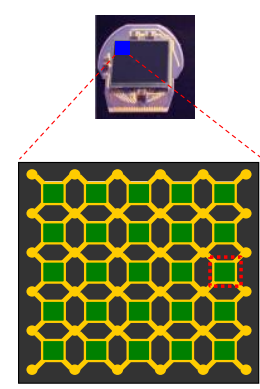

Fig. 1. (a) Cross section of optical and structural layers of MRG microspectrometer, (b) $3 D$ view of micromachined Fabry-Perot filter, and (c) potential array application of the technology 
Past work on the microspectrometer has focused on shortwave infrared (SWIR) and midwave infrared (MWIR) wavelength bands. For extension of this technology to imaging in the longwave infrared (LWIR) band, three issues need to be addressed:

1. Although Germanium is an ideal optical material to use in this band, silicon monoxide is far too absorbing to be of use. Therefore, the mirrors need to be redesigned using a less absorbing material. In this work, we use ZnS as a low-index non-absorbing medium for the mirrors, in place of the silicon monoxide. In addition to wideband transparency, Ge and ZnS also provide the advantage of high refractive index contrast (in the LWIR band, the refractive indices of $\mathrm{Ge}$ and $\mathrm{ZnS}$ are 4 and 2.2, respectively [3]).

2. Standard sapphire and Czochralski (CZ) silicon substrates are excessively absorbing in the LWIR band. As such, the substrates used for fabricating LWIR devices will need to be either germanium or float-zone (FZ) silicon.

3. A trade-off exists between the spectral resolution of the tuneable filter, and the signal-to-noise ratio (SNR) of the image. As the filter bandwidth is narrowed (resolution increased), the light throughput is reduced, resulting in a concomitant reduction in the SNR. As such, an optimal choice of filter bandwidth is required that allows sufficient multispectral discrimination, while also presenting sufficient light to the imaging array below. The performance requirements of a tuneable filter for LWIR imaging applications are summarized in table 1.

Table 1. Performance requirements of tuneable filter for LWIR imaging applications

\begin{tabular}{|l|c|l|}
\multicolumn{2}{|c|}{ Parameter } & \multicolumn{1}{c|}{ Value } \\
\hline Filter spectral width & $0.5-1 \mu \mathrm{m}$ & Optimization of multispectral vs SNR \\
\hline Peak transmission & $>50 \%$ & For low light conditions \\
\hline Out of band rejection & $>10: 1$ & Wavelength discrimination \\
\hline Wavelength tuning range & $8-12 \mu \mathrm{m}$ & Complete LWIR thermal band \\
\hline Tuning voltage & $<25 \mathrm{~V}$ & Compatibility with readout IC voltages \\
\hline
\end{tabular}

Due to the long infrared wavelengths considered, one of the key challenges in LWIR filter fabrication is the integration of top distributed Bragg reflector (DBR), which is usually composed of thick multi-layers, to achieve high reflectivity and create a sharp resonance. Multi-layer structures can give rise to significant stress mismatch that would highly deform the mirror and degrade filter performance. To combat these limitations, we have designed a Fabry-Perot filter comprised of a movable quarter-wave single-layer Ge top reflector and a fixed float-zone Si substrate incorporating a quarter-wave Ge-ZnS-Ge-ZnS bottom reflector, where the Ge and ZnS layer thicknesses are $625 \mathrm{~nm}$ and $1130 \mathrm{~nm}$, respectively. The filter layer structure is schematically presented in figure 2(a). Tuning spectra with variable air cavity length, $d$, are modeled using the transmission matrix method [4] and shown in figure 2(b). The proposed filter is capable of achieving bandwidths of about $0.5 \mu \mathrm{m}$, peak optical transmission higher than $85 \%$, and out-of-band rejection greater than 40:1 across the entire 8-12 $\mu \mathrm{m}$ LWIR wavelength band, which adequately fulfills the performance requirements of the tuneable filter for multi-spectral imaging applications listed in table 1.

(a)

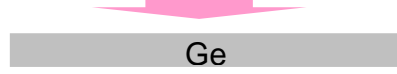

air cavity d

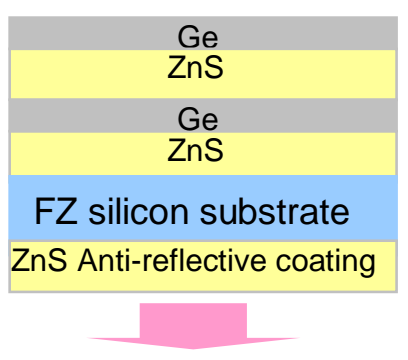

(b)

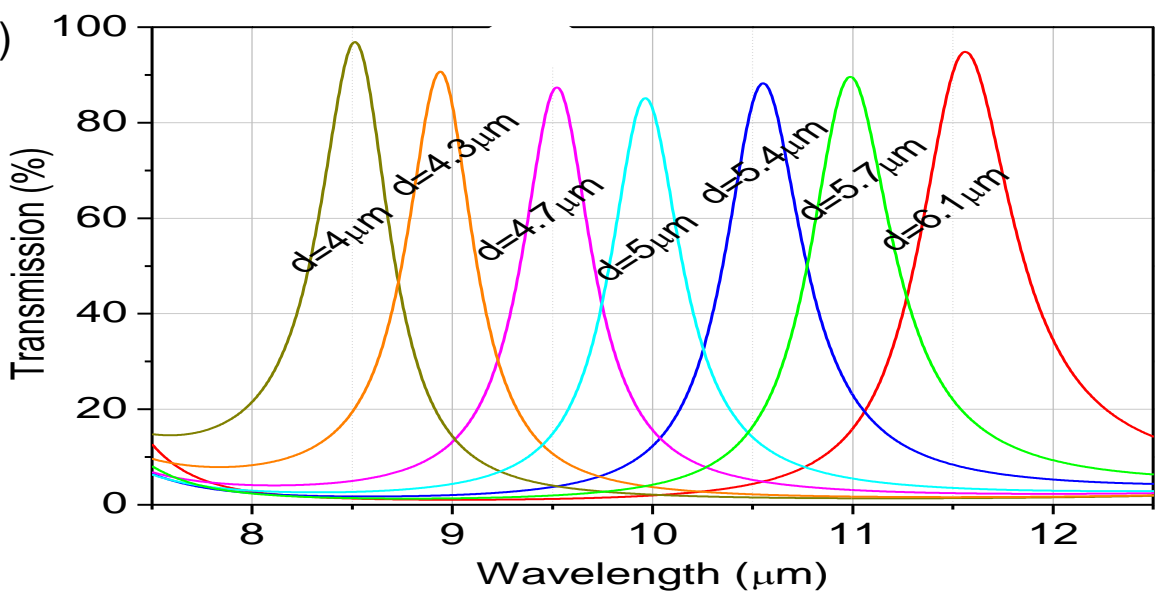

Fig. 2. (a) Schematic view of LWIR filter layer structure, and (b) the modeled tuning spectra of the filter

Another concern in designing filters for the LWIR band is electrostatic actuation. As indicated in figure 2(b), to tune the filter over the whole 8-12 $\mu \mathrm{m}$ band, doubly-clamped beam actuators will have to provide a displacement in excess of $2 \mu \mathrm{m}$ (optical cavity length change from above $6 \mu \mathrm{m}$ down to less than $4 \mu \mathrm{m}$ ). As shown in figure $3(\mathrm{a})$, simply employing the same actuator structure used for SWIR or MWIR filters would require a $6 \mu \mathrm{m}$ air gap between the top and bottom electrodes, which undoubtedly will incur an excessively high actuation voltage. To realize actuation using readout IC compatible voltages, a novel actuator design is proposed for LWIR filters, as shown in figure 3(b). In this design, an 
additional polyimide layer is introduced to raise the bottom electrode and decrease the actuation gap length and, as a consequence, reduce the actuation voltage. Meanwhile, the central portion of polyimide is selectively removed to maintain the same $6 \mu \mathrm{m}$ optical cavity length. This polyimide layer also functions as an insulation layer for the electrodes and prevents any leakage current flowing through to the semiconductor substrate.

(a)

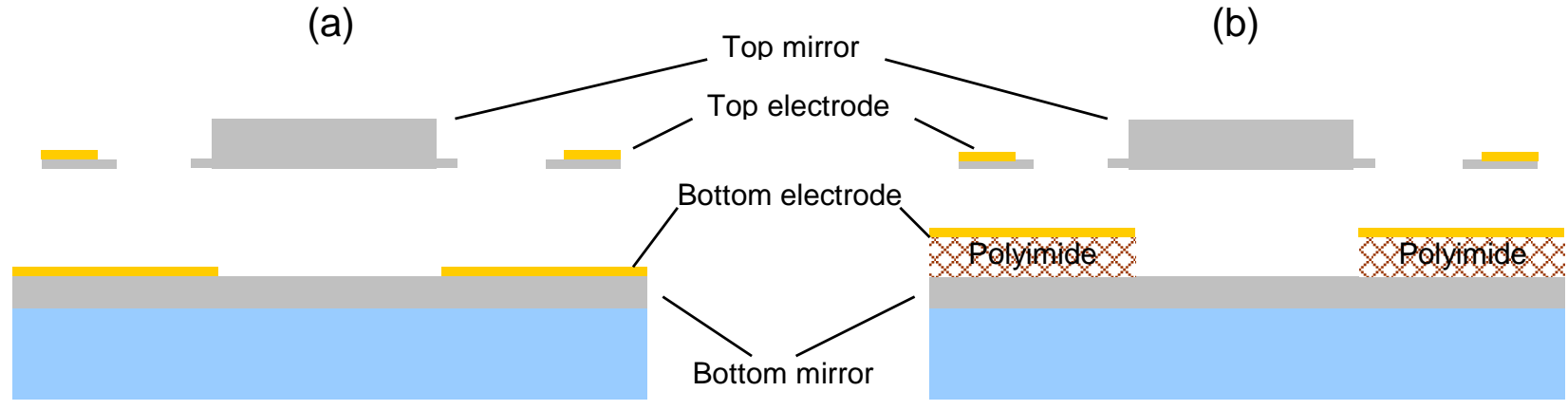

Fig. 3. Schematic view of actuator structure (a) in SWIR and MWIR filters and (b) in LWIR filters

Prior to fabrication, the deposition modality and parameters of the Ge and ZnS materials need to be established. It was found that durable Ge films could be deposited via E-beam at room temperature. Durable ZnS films could be deposited via thermally evaporation with the substrate held at $150^{\circ} \mathrm{C}$.

The fabrication process for LWIR filters is similar to that for SWIR and MWIR filters, as depicted in figure 4. First, a Ge-ZnS-Ge-ZnS (4-layer) quarter-wave bottom Bragg mirror is deposited on a float-zone silicon wafer, followed by the evaporation of another ZnS layer on the backside of the substrate as an anti-reflection coating (figure 4(a)). Polyimide is

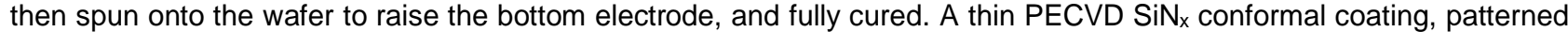
in $\mathrm{O}_{2} / \mathrm{CF}_{4}$ plasma, is used as a mask to protect all but the central portion of the polyimide from being etched in the $\mathrm{O}_{2}$ plasma used during the final release process. A $40 \mathrm{~nm}$ thick gold layer is then deposited and patterned using lift-off. The gold layer acts as the bottom electrode and also as an optical shield to block stray light from leaking around the filter onto the detector (figure 4(b)). Next, a sacrificial polyimide layer is spun on the wafer on top of the first polyimide, to form the target cavity thickness of $6 \mu \mathrm{m}$. Patterning of the polyimide to create step-down anchors to the substrate is accomplished by dry etching in $\mathrm{O}_{2} / \mathrm{CF}_{4}$ plasma. A compressively stressed PECVD $\mathrm{SiN}_{\mathrm{x}}$ layer is next deposited and patterned to balance the stress gradient in the top mirror (figure 4(c)). A $250 \mathrm{~nm}$ Ge structural layer is deposited and lift-off patterned to form the actuator beams and the mirror supports. Another E-beam evaporation and lift-off patterning of a $375 \mathrm{~nm} G e$ layer is made to complete the quarter-wave mirror. A two-stage Ge deposition is used to create actuation beams that are thinner than the quarter-wave mirror, effectively reducing the actuation voltage. Next, an array of holes of $4 \mu \mathrm{m}$ diameter are patterned through the mirror to facilitate the polyimide releasing process, allowing the mirror to be fully released within 1 hour and, therefore, preventing cracks forming in the Ge mirror due to overheating during any long time exposure to the $\mathrm{O}_{2}$ plasma (figure 4(d)). A $40 \mathrm{~nm}$ gold top electrode is then deposited and patterned on the beams using lift-off, followed by a thick gold deposition to strengthen the supports (figure 4(e)). Finally, the sacrificial polyimide layer forming the cavity is removed in an $\mathrm{O}_{2}$ plasma (figure 4(f)).

(a)

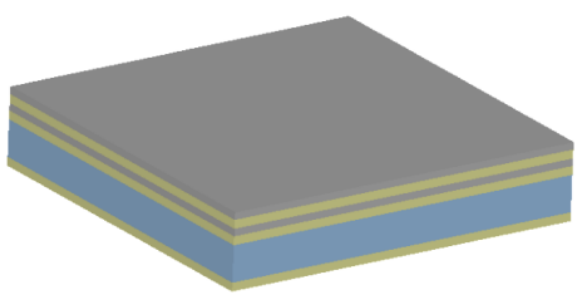

(b)

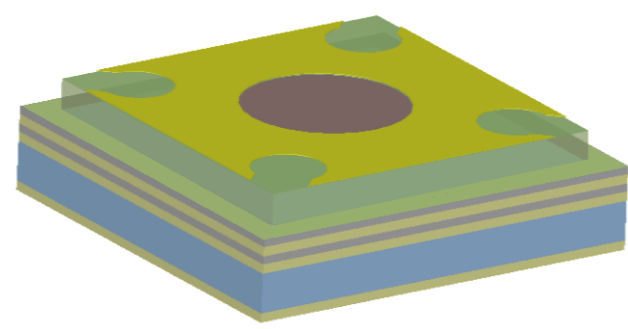


(c)

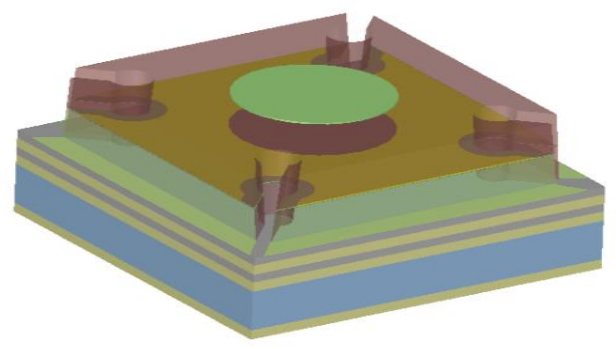

(e)

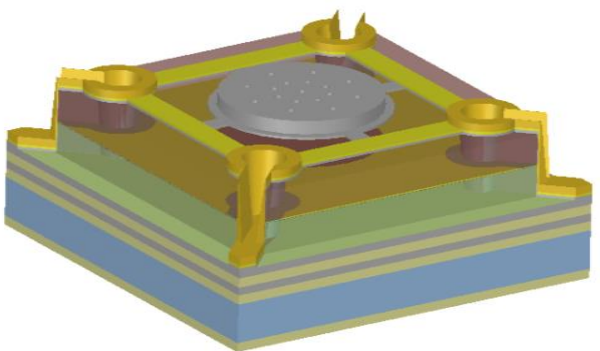

(d)

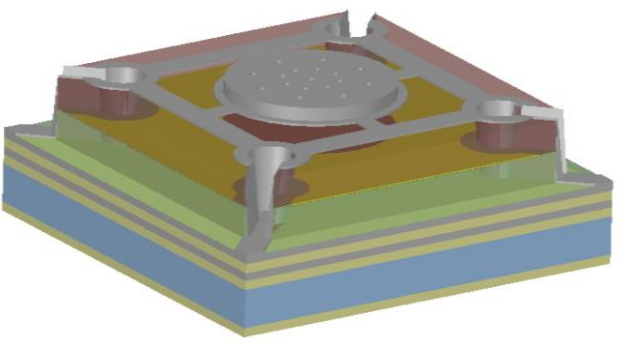

(f)

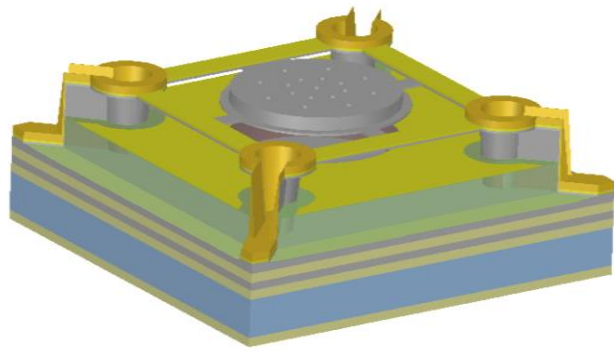

Fig. 4. Fabrication process flow of LWIR tunable Fabry-Perot filters

A scanning electron microscope (SEM) image with structural dimensions of the fabricated LWIR filter is shown in figure 5. In this design, the length of actuator beams and the mirror diameter are the same. For comparison purposes, devices with two different mirror diameters $(D=150 \mu \mathrm{m}$ and $200 \mu \mathrm{m})$ were fabricated. In order to characterize the dependence of actuation voltage on electrode separation, devices with $5.5 \mu \mathrm{m}$ and $3 \mu \mathrm{m}$ actuation gap length were fabricated by utilizing $0.5 \mu \mathrm{m}$ and $3 \mu \mathrm{m}$ thick lower polyimide layers, respectively.

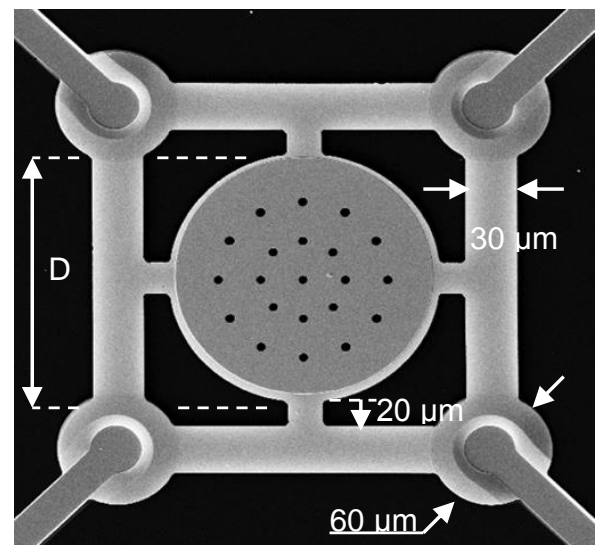

Fig. 5. SEM image of a fabricated LWIR filter and its structure dimensions

\section{Device characterization}

Whereas ideal Fabry-Perot filters have smooth, flat, parallel mirrors, the mirrors in a real filter exhibit imperfections due to surface/interface roughness, mirror tilt and mirror curvature, which tend to broaden the bandwidth and reduce the transmittance of the filter. While roughness and tilt can be eliminated by carefully handling the fabrication process, mirror curvature, which is essentially induced by a stress gradient profile within the mirror, needs additional effort to eliminate. It has been demonstrated in our SWIR and MWIR filters that a PECVD SiN layer as thin as $50 \mathrm{~nm}$ deposited underneath the Ge-SiO-Ge mirror can successfully be deployed as a stress compensator, and can be used to reduce the curvature of the mirror. Considering the highly absorbing nature of $\mathrm{SiN}_{\mathrm{x}}$ in the LWIR band, a much thinner $\mathrm{SiN}_{\mathrm{x}}$ layer must be used in order to ensure that no significant absorption is incurred, while also providing sufficient stress balancing. As such, all $\mathrm{SiN}_{\mathrm{x}}$ layers used must be highly stressed. In this work, $\mathrm{SiN}_{\mathrm{x}}$ with a predetermined compressive stress of $150 \mathrm{Mpa}$ was used. In order to determine the optimal thickness of the $\mathrm{SiN}_{\mathrm{x}}$ compensation layer, four samples were prepared with different deposition times of $0,14,21$ and $28 \mathrm{~s}$, corresponding to layer thickness of $0,4,6 \mathrm{and} 8 \mathrm{~nm}$, respectively. Both curvature, which is defined as reciprocal of radius of curvature, and center-to-edge bowing of released filters were measured using an optical profilometer, and the results are summarized in figure 6(a) and (b), respectively. 

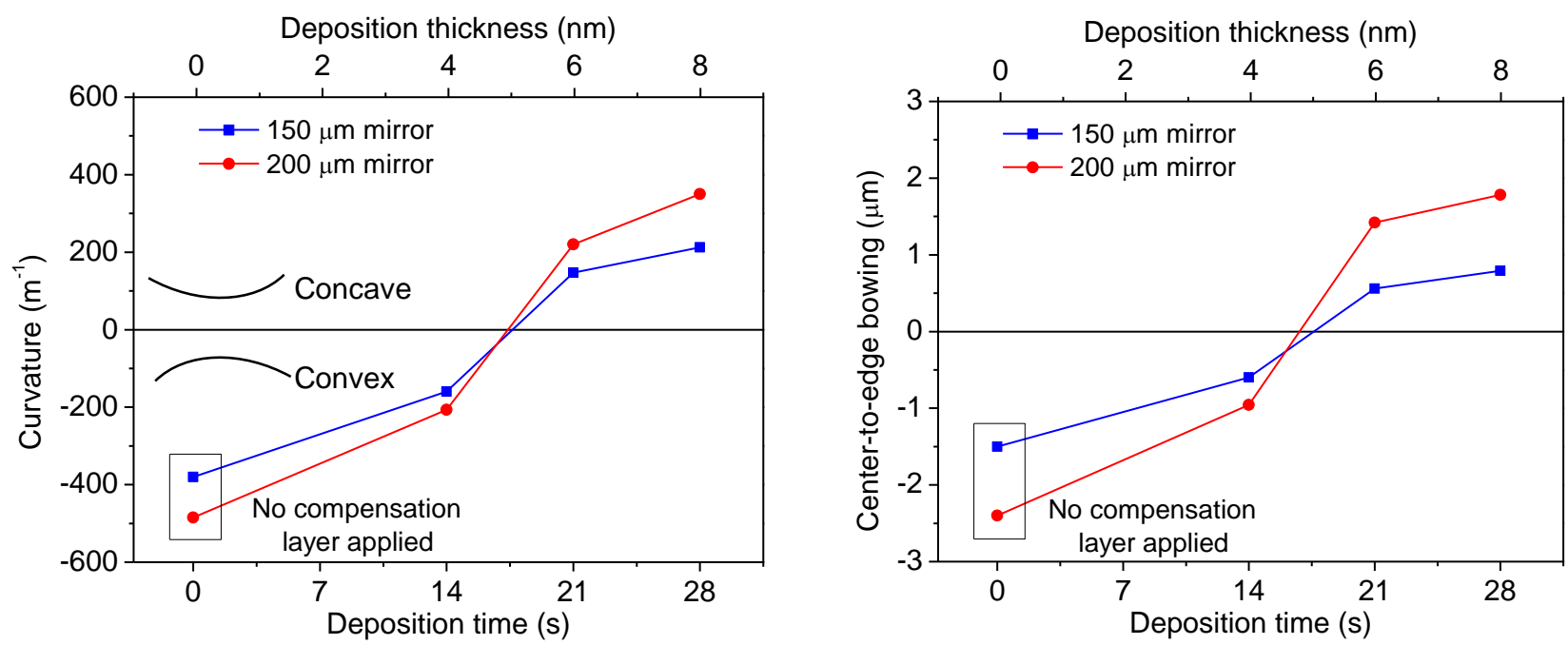

Fig. 6. (a) Curvature, and (b) center-to-edge bowing of released filter as a function of SiN $N_{x}$ compensating layer thickness. Curvature is defined as reciprocal of radius of curvature.

As seen from the data in figure 6(a), measurements taken immediately following fabrication of the filters with no compensation layer showed a curvature of $-380 \mathrm{~m}^{-1}$ and $-485 \mathrm{~m}^{-1}$, corresponding to a convex center-to-edge bowing of $1.5 \mu \mathrm{m}$ and $2.4 \mu \mathrm{m}$ for the $150 \mu \mathrm{m}$ and $200 \mu \mathrm{m}$ diameter mirrors, respectively. The convex shape indicates that the lower part of the Ge mirror is in a more tensile stress state than the upper part. There is a clear trend that with $4 \mathrm{~nm}$ thick $\mathrm{SiN} \times$ compensation layer deposited, the curvature is significantly reduced and moving towards zero; further deposition results in an abrupt change of curvature from convex to concave. Moreover, at the larger mirror diameter, the absolute curvature or center-to-edge bowing amplitude can be seen to be much greater than that for the smaller diameter mirror. It can be deduced that the larger mirror is more vulnerable to the stress gradient than the smaller mirror. A deposition time of approximately $17 \mathrm{~s}$, corresponding to a thickness of $5 \mathrm{~nm}$ was found to be optimal to achieve a zero curvature. Filter fabrication using a $17 \mathrm{~s}$ deposition time was undertaken. However, the outcomes were not as expected, resulting in a concave curvature of about $200 \mathrm{~m}^{-1}$ rather than the expected zero curvature. This is a clear indication that processing variations during deposition of the $\mathrm{SiN}_{\mathrm{x}}$ stress compensating layer need to be under better control before such a technology can be fully realized.
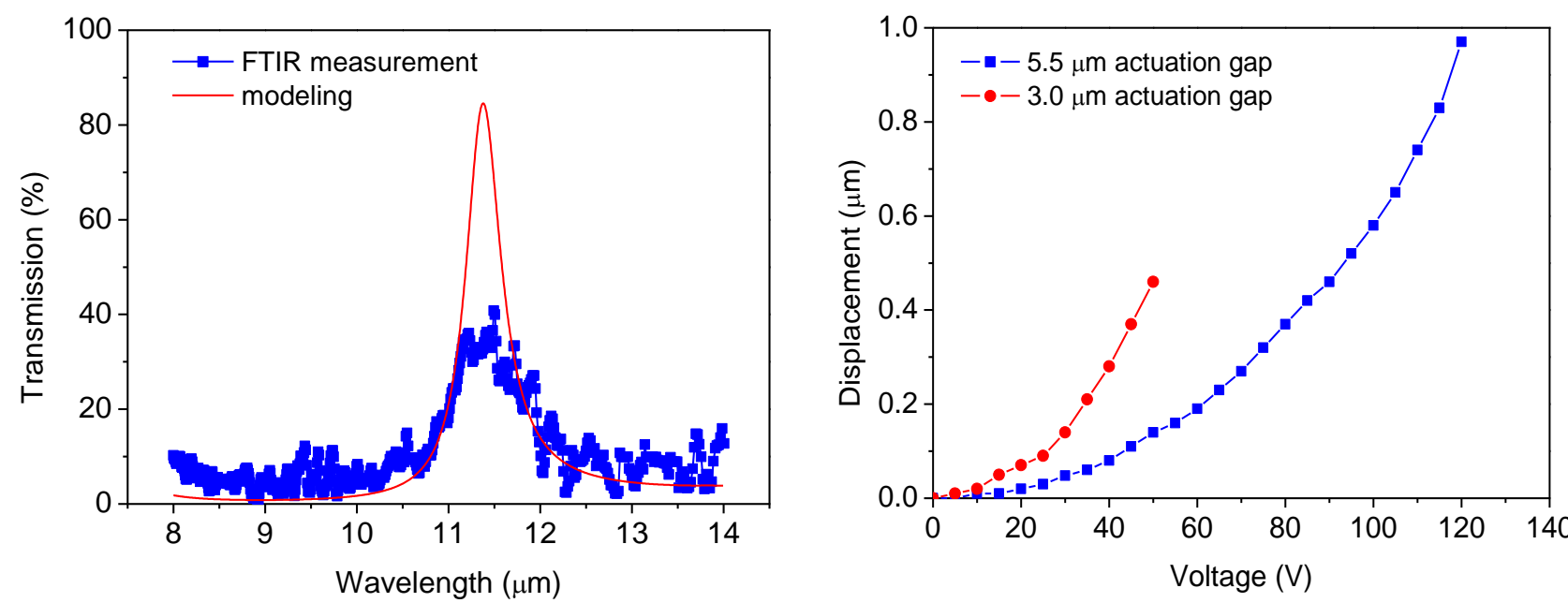

Fig. 7. (a) FTIR transmission measurement of fabricated $150 \mu \mathrm{m}$ diameter filter with $550 \mathrm{~nm}$ center-to-edge bowing, and (b) displacement of $200 \mu \mathrm{m}$ long doubly-clamped beam actuators

The optical transmittance of the $150 \mu \mathrm{m}$ diameter filter, which exhibited the least curvature of $-200 \mathrm{~m}^{-1} \mathrm{and} \mathrm{had}$ a convex center-to-edge bowing of $550 \mathrm{~nm}$, was measured using Fourier Transform Infrared Spectroscopy (FTIR). A reference transmission spectrum was first measured using a $150 \mathrm{um}$ opening in a $200 \mathrm{~nm}$ thick gold film on a silicon substrate. Then the sample was placed into the FTIR system and the transmission through the sample was measured. As evident from figure $7(\mathrm{a})$, the measured spectrum shows approximately $40 \%$ transmission at the peak wavelength, a $1 \mu \mathrm{m}$ FWHM spectral bandwidth, and an 8:1 out-of-band rejection. In comparison, 85\% peak transmission, $500 \mathrm{~nm}$ 
bandwidth, and 40:1 out-of-band rejection are predicted by the model for the ideal curvature-free filter. It is evident that the curvature in the mirror has severely degraded the spectral characteristics of the filter.

In a separate experiment, the physical displacement of the $200 \mu \mathrm{m}$ long double-clamped beam actuators comprising $5.5 \mu \mathrm{m}$ and $3 \mu \mathrm{m}$ actuation gaps were measured for different applied DC voltages, with the resultant voltagedisplacement curves being plotted in figure 7(b). For actuators comprising a $5.5 \mu \mathrm{m}$ actuation gap, 1 um of displacement was achieved with an actuation voltage of approximately $125 \mathrm{~V}$. Further increasing the actuation voltage generates large electric fields between the electrodes, leading to electrical breakdown of the air. Similarly, the maximum displacement achieved for actuators comprising a $3 \mu \mathrm{m}$ actuation gap was $0.5 \mu \mathrm{m}$ at $50 \mathrm{~V}$ applied bias, above which electrical breakdown also occurred. As evident from figure 7(b), the beam actuator with a narrower actuation gap requires a lower actuation voltage to achieve the same displacement in comparison to a device with a wider actuation gap. While neither device achieved the required $2 \mu \mathrm{m}$ displacement before electrical breakdown, this work has presented some significant steps that have been achieved towards realising surface micromachined tuneable filters operating in the LWIR spectral band.

\section{Conclusions and future work}

This paper reports on our preliminary investigations towards extending our mature SWIR and MWIR microspectrometer technology to the LWIR wavelength band. The proposed LWIR filter design shows promise in being able to achieve the performance requirements of multi-spectral imaging applications, and to realize tuning across the full range of the LWIR band using low voltage actuation. Whereas the fabricated prototype filters only achieved $40 \%$ peak transmission, an 8:1 out-of-band rejection ratio, and a $1 \mu \mathrm{m}$ bandwidth with actuator displacement at voltages that are too high to be compatible with readout ICs, we are working on design modifications to overcome these limitations. Future work will focus on finding an optimized $\mathrm{SiN}_{\mathrm{x}}$ deposition process that will not cause abrupt changes to the mirror curvature, and that will form the basis of a reliable and repeatable stress compensation layer. Extending the length of the beam actuators will be examined as an option to limit the actuation voltage to within the readout IC compatibility range. However, as the length of the beam actuators is increased, curvature elimination in the resultant larger diameter mirror becomes more difficult. This trade-off needs to be carefully considered and addressed.

\section{REFERENCES}

[1] Milne J. S., Dell J. M., Keating A. J., Faraone L., "Widely Tunable MEMS-Based Fabry-Perot Filter”, J. MEMS, 18(4), pp 905 - 913, 2009.

[2] Keating A. J., Antoszewski J., Silva K. K. M. B. D., Winchester K. J., Nguyen T., Dell J. M., Musca C. A, Faraone L.,Mitra P., Beck J. D., Skokan M. R.,Robinson J. E., "Design and Characterization of Fabry-Perot MEMS-Based Short-Wave Infrared Microspectrometers", J. Elec. Mat., 37(12), pp. 1811 - 1820, 2008.

[3] Edward. D. P., "Handbook of Optical Constants", Academic Press, 1998.

[4] Hugh. A. M., "Thin-film Optical Filters", Institute of Physics Publishing, 2001. 